\title{
Exploring quality of life in families of children living with and without a severe food allergy
}

\section{Althea Z. Valentine and Rebecca C. Knibb}

\section{Introduction}

Food allergy (FA) has been shown to affect quality of life (QoL) in both adults (Primeau et al., 2000) and children and teenagers (Avery et al., 2003, Cummings et al., 2010a, Cummings et al., 2010b and Sicherer et al., 2001). QoL can be defined as well-being which is determined by both objective and subjective factors, across a number of life domains which are deemed as important in one's culture and time (Wallander, 2001). Over the past 20 years, there has been a growing body of research looking at QoL in children and research indicates that children as young as 5 years of age are able to respond to QoL questionnaires (Jirojanakul \& Skevington, 2000).

A number of child QoL measures have been developed in both generic and disease specific forms, which allow children to rate their own QoL (see Eiser \& Morse, 2001). FA specific QoL measures have recently been developed (e.g. DunnGalvin, Flokstra-de Blok, Burks, Dubois, \& Hourihane, 2008) and comparisons with generic health related QoL measures have shown them to be more sensitive to clinically important differences for food allergic patients (Flokstra-de Blok et al., 2010a). Generic questionnaires, however, allow general QoL to be examined and comparisons to be made across groups of people, healthy individuals and across different diseases. Flokstra-de Blok et al. (2010b) compared generic health related QoL of children, adolescents and adults with food allergy to that of a healthy population and other diseases. They found that those with FA reported greater limitations on social activities and poorer overall health than the general population and poorer health related QoL than patients with diabetes mellitus.

There has been relatively less research looking at QoL in families living with FA. The impact of a chronic illness on family members varies greatly between families, but it is clear that the family plays a pivotal role in determining how children with chronic conditions adapt to their condition and how it impacts upon their QoL (Hanson, 2001). It is generally accepted that children with chronic conditions potentially have lower QoL because of the additional demands and stressors placed on children by their condition (Hanson). In the case of FA, like many other chronic conditions, parental QoL may be affected by the child's illness, as families have to deal with the day-to-day management and emotional strain of the illness, as well as additional costs in terms of time and money and disruption to everyday household routines (Sales, 2003). Arvola, Tahvanainen, and Isolauri (2000) report that over $90 \%$ of parents found caring for a child with atopic conditions more demanding and stressful than a healthy child, mainly because of skin treatments (associated with atopic illnesses such as eczema), pruritus, restlessness, lack of sleep, and adherence to an elimination diet. These authors suggest, to improve care and support for parents with atopic infants, it is necessary to identify and understand parents' concerns.

Research looking specifically at FA and QoL suggests that QoL may be affected by: having to remain constantly vigilant about food eaten; social restrictions, for example not being able to eat school dinners and not being able to share foods at parties (Gowland, 2002); greater daily disruption of activities and more difficulties in family social interactions (Primeau et al., 2000); and through decreased general health perceptions, parental emotional distress, and limitation on family activities (Sicherer et al., 2001). However, these studies were restricted because the child's QoL was assessed by proxy ratings and some used very different comparison groups. For example, QoL in children with rheumatological diseases (Primeau et al., 2000) may be influenced by pain, fatigue, and physical limitations; factors which are not generally pertinent to those with allergies.

More recently studies have asked both children and parents to complete their own generic and FA specific QoL scales and have found that mothers suffer greater anxiety and stress than fathers, and children with FA suffer poorer QoL than their siblings (King, Knibb, \& Hourihane, 2009). Cummings et al. (2010) also found high anxiety levels in 
mothers and poorer QoL in children with nut allergies compared to healthy population norms and Springston et al. (2010) reported that parents had greater social limitations resulting from their child's FA. All of these studies used quantitative methodology to gather data. Mixed designs which incorporate qualitative methodology have the potential to gather richer, more in depth information about the impact of FA on the child and family.

Avery et al. (2003) examined QoL from the perspective of 20 children with peanut allergies compared to 20 children with diabetes mellitus. Using QoL measures designed or adapted for the study, the authors reported significantly poorer QoL in children with peanut allergies compared to those with diabetes mellitus. The second part of this study involved children recording how their condition impacted upon their QoL by taking photographs and commenting in notebooks; a method sometimes called 'Photovoice'. This study can be commended for its innovative methodology which resulted in a number of themes children felt were important regarding their peanut allergy, including food management, the environment, restaurants and other people. However, only one significant difference was produced in the 'Photovoice' part of their study, with peanut allergic children taking more restaurant related photographs; this may have occurred because children were restricted to taking photographs within a 24-h period at the weekend.

The present study aimed to expand on previous research by adopting the mixed methodology approach used by Avery et al. (2003) and additionally assessing parental QoL, using validated QoL measures, using children with a range of food allergies, extending the duration of the study to one week (including school), and utilising a comparison group of healthy children. Children with severe allergies to any foods were recruited, as it was considered important to identify how allergies affect all children to allow greater generalisation of results. A control group of healthy children was selected as a comparison group, as most children with FA are educated in mainstream schools and so FA children, like children with many other chronic conditions, are likely to compare themselves to what healthy children can do, rather than what other children with chronic conditions can do (Hourihane, 2001).

The aim of this study was to explore QoL in FA children in comparison to healthy control children and their respective primary caregivers, using generic QoL measures and a 'Photovoice' method.

\section{Methods}

\section{Participants}

FA families were recruited via the Anaphylaxis Campaign and had one or more children aged 8-12 years with a clinically confirmed IgE mediated FA (by skin prick test or food challenge in clinic). Participants with any level of FA severity were recruited, but, $34(100 \%)$ of FA child participants had prescribed adrenaline auto injectors and were at risk of anaphylaxis. Control families were recruited via advertisements within Universities in the East Midlands and out of school clubs. They consisted of children aged 8-12 years who had no chronic illnesses or disabilities in their immediate family. Families with additional atopic conditions within the family were allowed to participate in the study. It is appreciated that additional atopic conditions may also impact on QoL, but due to the high rate of comorbidity both within individuals and within families, inclusion criteria allowed these conditions to be present. Families with any other physical/mental illnesses or disabilities were excluded as these may have also had an impact on families' QoL.

\section{Materials}

Families were given a project pack containing child and adult information booklets; questionnaires; structured photograph diaries; consent letter to take photographs in school; return envelopes and disposable cameras ( 27 exposure) with flash.

A bespoke questionnaire gathered demographic and health information. Parents were asked if they or their child suffered from any chronic illness. In the FA condition, primary caregivers were additionally asked to state how their child's food allergy had been diagnosed and rate the severity of their child's FA on a four point scale (mild, moderate, 
severe, life threatening). Parents were also asked to indicate whether their child carried an adrenaline auto injector, and to describe the food(s) their child was allergic to and his/her symptoms.

QoL in adults was assessed using the WHOQOL-BREF (WHOQOL Group, 1996), a shortened 26-item version of the WHOQOL-100 assessment (WHOQOL Group, 1998). The WHOQOL-BREF scale has good to excellent internal consistency (reliability) and adequate discriminant and construct validity (Skevington, Lotfy, \& O'Connell, 2004). All items were rated on a five point Likert scale and asked about life in the last two weeks. Data was recoded according to the scoring guidelines (WHOQOL Group, 1996) in order to acquire four domain scores: physical health, psychological well-being, social, and environmental factors. Two individual items looking at overall QoL and satisfaction with health were also analysed. A higher score indicates a better quality of life.

Children's QoL was measured using a generic children's QoL questionnaire - the PedsQL ${ }^{\mathrm{TM}}$. Validity and reliability of this version have previously been assessed in a field trial of children with and without chronic conditions (Varni, Seid, \& Kurtin, 2001). The PedsQL is a 23-item self-report measure that takes approximately 4 min to complete and considers physical, emotional, social, and school functioning, as well as psychosocial health. All items are rated on a five point Likert scale and ask about QoL in the past one-month. Results were recoded following the scoring guidelines (Varni, 2004). Each summary score ranged from 0 to 100, with a higher score indicating a better QoL. Permission to make minor amendments to the wording of four items, to create a UK version of the questionnaire, was obtained from the test publishers. For example, "It is hard for me to walk more than one block" was changed to "It is hard for me to walk down the road a little bit". Generic rather than disease specific QoL of life scales were selected to allow for direct comparisons with the healthy control group.

\section{Ethics}

Ethical approval was obtained from the Psychology Research Ethics Committee at the University of Derby. Parents provided written consent for their family's participation prior to inclusion in the study. Adult information and questionnaire booklets included a check box for participants to opt out of having their photographs or quotes used in publications. It was also stressed that photographs of people or recognisable places would not be used in publications, to ensure anonymity of participants. Additionally, participants were instructed that they must obtain prior consent from all people that they photographed. Children who wanted to take photographs at school, and who conducted the study during term time, obtained signed consent from the head teacher. If head teachers refused to allow photographs to be taken at school, or if children conducted the study during school holidays, children were encouraged to take photographs of items relating to school, such as their bag or uniform.

\section{Procedure}

Focus groups with 20 children aged 7-11 years were initially run to develop age-appropriate definitions of QoL. All materials were piloted on 5 children aged between 8 and 12 years old to ensure that they could understand all instructions and knew how to use the cameras and record in their diaries. A pilot study with two families was then completed and minor amendments made before commencing the main study.

Participants were directly given or sent project packs via the postal system. First, participants completed the QoL questionnaire and then took photographs for a period of one-week and recorded details of these in a diary format. The structured diary format guided adult participants to state what they had taken a photograph of and how this influenced their QoL. Children were asked to complete two sentences that began, "This is a photo of..." and "I took this photo because it made me feel...". Parents then returned the packs by post.

\section{Data transcription and analysis}

Questionnaire data was inputted into SPSS (Version 16.0, SPSS Inc., Chicago, IL, USA). Independent Samples T-tests, One-Way ANOVAs and Mann-Whitney tests were used, as appropriate, to analyse the questionnaire data. A twotailed significance level of less than 0.05 was considered significant in all analysis. 
Photographs and diary text were subjected to content analysis. A coding scheme was developed with explicit category definitions and coding rules to avoid ambiguity and to ensure that categories were independent, mutually exclusive, and exhaustive (Neuendorf, 2001). In line with previous research (Avery et al., 2003), items were then rated using participant's diary comments to make latent inferences about how their QoL was affected. All items were allocated an arbitrary value from one to four depending on how it influenced QoL: 1 = positive influence, $2=$ negative influence, 3 = neutral statement/if it was not clear how it influenced QoL/or if items did not have a corresponding statement and $4=$ both positive and negative factors (mixed). Saturation point was reached after coding $76 \%$ of participants' diaries and photographs and no new codes emerged after this point.

\section{Results}

90 completed or part completed packs were returned, a response rate of $59 \%$ in FA families and $44 \%$ in non-FA families. The sample included: 43 adults ( $95 \%$ female), 34 children with a clinically confirmed IgE mediated FA (17 boys and 17 girls, mean age $=9$ years, SD $=1.22$ ) and 15 non-FA-control children ( 6 boys and 9 girls, mean age $=9$ years, $S D=1.01)$. All children were white British $(91 \%)$ or mixed heritage $(9 \%)$. Further FA child details are recorded in Table 1.

Table 1. - Details of age of onset, symptoms and severity of allergy in FA child participants.

\begin{tabular}{|c|c|c|}
\hline & \multicolumn{2}{|c|}{ Number and percentage of participants } \\
\hline & $N$ & $\%$ \\
\hline \multicolumn{3}{|l|}{ Age of diagnosis } \\
\hline $0-12$ months & 14 & 41.2 \\
\hline 13-24 months & 9 & 26.5 \\
\hline 25 months+ & 9 & 26.5 \\
\hline Undisclosed & 2 & 5.8 \\
\hline \multicolumn{3}{|c|}{ Parental rating of severity of allergy } \\
\hline Life threatening & 23 & 67.6 \\
\hline Life threatening/severe & 2 & 5.9 \\
\hline Severe & 5 & 14.7 \\
\hline Moderate & $1^{\mathrm{a}}$ & 2.9 \\
\hline Mild & 0 & 0 \\
\hline Undisclosed & 3 & 8.8 \\
\hline \multicolumn{3}{|l|}{ Foods allergic to ${ }^{b, c}$} \\
\hline Peanuts & 18 & 52.9 \\
\hline Multiple nuts & 27 & 79.4 \\
\hline Eggs & 7 & 20.6 \\
\hline Dairy & 3 & 8.8 \\
\hline Fruit and vegetables & 8 & 23.5 \\
\hline Multiple foods & 24 & 70.6 \\
\hline Others (e.g. seeds) & 7 & 20.6 \\
\hline \multicolumn{3}{|l|}{ Symptoms ${ }^{d}$} \\
\hline Breathing difficulties & 5 & 14.7 \\
\hline Swelling & 13 & 38.2 \\
\hline Rash & 16 & 47.1 \\
\hline Itching & 8 & 23.5 \\
\hline Wheezing & 10 & 29.4 \\
\hline Vomiting/diarrhoea & 11 & 32.3 \\
\hline Anaphylaxis & 14 & 41.2 \\
\hline Others (e.g. runny nose) & 9 & 26.5 \\
\hline
\end{tabular}


a. This parent also acknowledged that his/her doctor believed that the child's allergy was life threatening.

b. Six children had additional (non-food) allergies including: grass, latex, animals, trees, and dust mites.

c. Many children had allergies to multiple foods and numerous symptoms, hence percentages total greater than $100 \%$ in the 'foods allergic to' and 'symptoms' rows.

d. Four parents did not disclose specific symptoms.

\section{Primary caregiver's QoL}

Complete WHOQOL-BREF data was received from 42 parents (13 non-FA-control, 29 FA). No significant differences in demographics were found between the two groups. Both FA and control parents' ratings were similar across most domains, although FA parents scored lower than control parents for social relationships. Similarly, FA parents' ratings of overall QoL were significantly lower than the non-FA-control parents' (Table 2).

Table 2. - Comparison of mean WHOQOL-BREF QoL scores in parents with and without FA children, and mean PedsQL ${ }^{\mathrm{TM}}$ scores for each QoL domain, for children with and without a food allergy.

\begin{tabular}{|c|c|c|c|c|c|}
\hline \multirow[t]{2}{*}{ WHOQOL-BREF } & \multicolumn{2}{|c|}{$\begin{array}{l}\text { FA parents } \\
(N=29)\end{array}$} & \multicolumn{2}{|c|}{$\begin{array}{l}\text { Control parents } \\
(N=13)\end{array}$} & \multirow[t]{2}{*}{$p$-Value } \\
\hline & Mean & SD & Mean & SD & \\
\hline Physical health & 17.16 & 1.89 & 17.98 & 1.18 & $t=-1.43$ \\
\hline Psychological & 15.68 & 1.45 & 15.79 & 1.34 & $t=-0.25$ \\
\hline Social relationships & 14.48 & 3.16 & 16.51 & 2.70 & $t=-2.01^{\circ}$ \\
\hline Environment & 15.76 & 1.72 & 15.50 & 1.50 & $t=0.47$ \\
\hline QoL score & 4.07 & 0.59 & 4.54 & 0.52 & $U=114.0$ \\
\hline Satisfaction with health & 4.00 & 0.80 & 4.31 & 0.63 & $U=150.0$ \\
\hline \multirow[t]{2}{*}{ PEDS-QL } & \multicolumn{2}{|c|}{$\begin{array}{l}\text { FA children } \\
(N=14)\end{array}$} & \multicolumn{2}{|c|}{$\begin{array}{l}\text { Control children } \\
(N=34)\end{array}$} & $p$-Value \\
\hline & Mean & SD & Mean & SD & \\
\hline Physical functioning & 93.66 & 7.40 & 87.05 & 10.24 & $t=2.51^{\circ}$ \\
\hline Emotional functioning & 70.15 & 14.54 & 70.71 & 14.79 & $t=-0.12$ \\
\hline Social functioning & 83.82 & 15.43 & 82.86 & 14.51 & $t=0.20$ \\
\hline School functioning & 80.29 & 14.51 & 76.07 & 16.89 & $t=0.87$ \\
\hline Psychosocial health & 78.09 & 11.78 & 76.55 & 13.45 & $t=0.40$ \\
\hline Total QoL score & 83.50 & 8.85 & 80.20 & 11.42 & $t=1.08$ \\
\hline
\end{tabular}

\section{Children's QoL}

48 children completed the PedsQL ${ }^{\mathrm{TM}}$ (14 control and $34 \mathrm{FA}$ ). No significant differences were found between demographic variables for FA and healthy children. FA children had similar or higher QoL scores than control children across all domains (Table 2). The only significant difference was in the physical functioning domain, where FA children reported better functioning than control children.

\section{'Photovoice' analysis}

Diaries and cameras were returned from 29 FA parents, 33 FA children, 10 comparison parents, and 14 comparison children.

The coding process reduced the 'Photovoice' data (photographs and diary entries) to items that could be coded using content analysis. A random sample of $30 \%$ of the photographs and corresponding text were selected using a random number table. An independent researcher then coded this subsection of data, using the coding manual and 
coding guidelines. Inter-coder reliability was high, researchers agreed on $89 \%$ of the adult codes and $97 \%$ of the child codes, an overall agreement of 93\% (Kappa $r=0.91, p<0.01$ ). Additionally, each item was rated in terms of how it influenced QoL. Inter-coder reliability of this was also high, with $96 \%$ agreement (Kappa $=0.92, p<0.01)$. Where researchers disagreed, they discussed each item until $100 \%$ consensus was reached and modifications were made to the coding manual accordingly. FA parents and children had slightly more negative, neutral, and mixed rated codes, and slightly fewer positive codes, than control parents and children respectively (see Table 3 ). The difference in the number of positive codes between FA (mean $=8.24, S D=5.45$ ) and control parents ( $m e a n=12.50, S D=6.54$ ) was significant, $(p=0.05)$. Similarly, FA children had significantly more neutral codes (mean $=2.55, S D=3.88)$ than the control children (mean $=0.86, \mathrm{SD}=1.10),(p=0.027)$.

Table 3. - Percentage of participants with codes in each category, for children with and without food allergies and their respective primary caregivers. [table unavailable in this version]

From the coding process, ten themes emerged that were found to influence QoL across conditions these were: activities of daily living, education/employment, environment, food/drink, health, home/garden, leisure activities, morals/values/spirituality, people, and places. As seen in Table 3, participants across all conditions recorded some categories (food/drink, home/garden, leisure, places, and people) as important positive determinants of QoL. In particular, family and social support were commonly referred to across conditions. However, Table 3 and Table 4 also clearly show quantitative differences in a number of categories, which are discussed further here.

Table 4. - Comparison of the mean and standard deviation of the total number of codes within each QoL domain, for children with and without food allergies and their respective primary caregivers.

\begin{tabular}{|c|c|c|c|c|c|c|c|c|}
\hline & \multicolumn{4}{|c|}{ Primary caregivers } & \multicolumn{4}{|c|}{ Children } \\
\hline & \multicolumn{2}{|c|}{ Allergy $(N=29)$} & \multicolumn{2}{|c|}{ Control $(N=10)$} & \multicolumn{2}{|c|}{ Alergy $(N=33)$} & \multicolumn{2}{|c|}{ Control $(N=14)$} \\
\hline & Mean & SD & Mean & SD & Mean & SD & Mean & SD \\
\hline Activities of daily living & $0.59^{\circ}$ & 0.78 & $2.40^{\circ}$ & 2.07 & 0.30 & 0.64 & 0.79 & 1.25 \\
\hline Education and employment & 1.48 & 1.70 & 1.20 & 1.23 & $1.52^{\circ}$ & 1.70 & $0.71^{\circ}$ & 0.91 \\
\hline Environment & 0.45 & 1.21 & 2.10 & 2.56 & 0.45 & 0.97 & 0.50 & 0.65 \\
\hline Food and drink & $3.93^{\circ}$ & 3.57 & $0.80^{\circ}$ & 0.92 & $3.30^{\circ}$ & 3.34 & $1.29^{\circ}$ & 1.54 \\
\hline Health & $2.72^{\circ}$ & 2.49 & $0.40^{\circ}$ & 0.52 & $2.00^{\circ}$ & 3.45 & $0.14^{\circ}$ & 0.36 \\
\hline Home and garden & 1.83 & 1.28 & 3.30 & 2.63 & 1.73 & 1.31 & 2.93 & 3.39 \\
\hline Leisure activities & 1.72 & 1.79 & 2.60 & 1.84 & $3.09^{\circ}$ & 2.56 & $5.36^{\circ}$ & 1.99 \\
\hline Morals, values and spirituality & 0.07 & 0.26 & 0.20 & 0.42 & 0.12 & 0.33 & 0.36 & 0.75 \\
\hline People & 2.97 & 2.41 & 3.60 & 2.32 & 4.15 & 3.57 & 3.43 & 1.83 \\
\hline Places & 1.66 & 2.09 & 1.20 & 0.92 & 1.45 & 2.03 & 1.43 & 1.51 \\
\hline
\end{tabular}

\section{Activities of daily living}

Significantly more control parents had codes for activities of daily living than allergy parents (Table 4). Many control parents' codes were positive. For example, codes related to time and energy saving appliances such as dishwashers. Photographs of telephones were also popular positive influences on QoL, as were personal care activities such as taking a bath, putting on make up and going to a beauty salon. The most referred to negative influence on QoL in this category was ironing. In contrast, FA parents' codes were more evenly split across positive and negative domains (Table 3). These mainly reflected additional household chores relating to FA (and associated atopic conditions), for example, "constantly washing to remove dust mites - in order to protect my children from 'extra' itches".

\section{Education and employment}

In this category photographs mainly focused on schools. Both FA and non-FA children reported liking and disliking various aspects of school. However, FA children had significantly more codes in the education/employment category than control children (Table 4). A number of these codes were allergy related, referring to support from peers and staff, and school lunches. School lunches were a particular issue with both FA parents and children, as illustrated by 
the following parental quote: "this makes me feel quite sad and powerless if I am honest. (Name of child) is not able to stay for school dinners because they cannot guarantee that the food is nut free... He has to sit in a different area of the dining hall to eat his packed lunch often away from his friends... He feels alienated because of this".

FA parents reported that their QoL was improved through helpful and supportive staff at their child's school, but was limited by lack of understanding from teachers. In contrast, non-FA parents reported that employment issues such as redundancy, long shifts and pension fears negatively influenced their QoL. Additional codes in this category were similar across FA and non-FA families and included positive codes referring to enjoyment of work for financial reward and independence, as well as parents proudly reporting their child's achievements at school.

\section{Food and drink}

Unsurprisingly, this was one of the largest categories (see Table 3) and 85\% of FA families took at least one food related photograph, in comparison to only $58 \%$ of control families. As seen in Table 4 , both FA parents and FA children had significantly more codes in this category than their respective control counterparts.

Positive comments in both groups referred to liking products, others reflected the positive aspects of family company during meal times and an opportunity to talk with family members. Only one healthy control parent commented how much they enjoyed eating out because they "don't have to cook". Whereas eating out was a more popular topic for FA parents, for whom eating out influenced their QoL in different ways. Some were positive, for example, "...just after a delicious lunch at an award-winning fish and chip restaurant (where we know the food is safe for (name of child) so none of us are anxious about the food ingredients and we don't have to press the staff for details)". However, most FA families' codes were negative for reasons of anxiety and fear about eating out, as well as feeling left out from social events. For example, "Our friend was having a barbecue two other families were invited but we weren't. I was told it was because my daughter is too difficult to cater for. This made me very angry and upset. I didn't tell my children...".

Supermarkets received favourable comments from non-FA parents whereas FA parents' comments were mainly negative and reflected the frustration they felt having to make extra shopping trips or go to multiple supermarkets to complete their weekly shop. One adult stated: "all these yummy cakes in the supermarket have a sign in front 'May Contain Nuts', etc. It makes me quite sad because surely not everything may be contaminated - but we can't take the risk". Labelling both limited and improved FA participant's QoL. For example, one child stated that they felt, "Happy because I can trust a product because of the packaging: nut free".

A few FA families did reflect on some positive factors about living with a FA, commenting that their diet was healthier or broader as a result.

\section{Health}

A large number of FA families (84\%) had health related codes compared to control participants (58\%; Table 3 ). As seen in Table 4, the mean number of health related codes was significantly higher in both FA adults and FA children, in comparison to their respective non-FA controls.

It was apparent that healthcare service provision varied across the country and this had an effect on QoL as evidenced by the photographs taken. In particular, lack of specialised allergy services, being given incorrect prescriptions, and healthcare professionals not knowing to check for medic-alert bracelets were highlighted as areas of concern. Many codes related to allergy medication and most comments from children were about carrying his/her adrenaline pens. One child reported that these made her feel 'a bit scared...and responsible because you feel grown up to look after yourself', whilst another reported that it made her feel 'safe'. Several children and parents had mixed feelings about their medications, as one child expressed, "This made me... feel sad. It also makes me feel happy because if I have a elergic [allergic] reaction the medcin [medicine] can help me". 
Some FA parents reported negative emotions about his/her child's food allergies, such as worry and guilt and hope that their child will grow out of the allergy. However, not all participants felt emotionally involved. Some participants tried to gain a sense of normality and accepted their child's allergy as just one of many concerns associated with being a parent.

\section{Leisure}

A large proportion of children across both conditions ( $F A=91 \%$, control $=100 \%$ ) had codes in this category. Although children in the control group had significantly more codes than the FA children, similar codes were evident across both conditions, the majority of which were positive influences on QoL (Table 3). Only two codes related to allergy. One child referred to playing on gaming equipment whilst waiting at hospital, another took a photograph of golf clubs and commented that they made him feel, "happy because it shows that I can still play sport". This may imply that he or other people cannot play sport because of chronic illness or alternatively that the child is restricted in a number of ways because of his allergy, but at least he can still play sport.

\section{Discussion}

The present study explored QoL in children (aged 8-12) with and without severe food allergies and their respective primary caregivers. Questionnaire analysis showed that FA parents reported poorer social relationships, than non-FA control parents. This was subsequently confirmed in the 'Photovoice' analysis where parents identified family and social support as one of the key determinants of QoL. Previous research has suggested that a child's QoL may be impaired by restrictions on social activities, for example, when going to parties (Gowland, 2002), and in family social interactions (Primeau et al., 2000). However, little research has considered the impact socially for the parent. This study adds to the allergology literature and draws attention to the need to consider the family as a whole, rather than simply focus on the FA child, when assessing QoL. The present study also found that FA parents scored significantly lower than control parents on the item asking about overall QoL, however, due to the design of the study, replication of this finding is needed as the number of participants is small resulting in low power. It has also been argued that the item "How would you rate your overall QoL" is too vague and non-specific to be used by itself, but may be a useful addition to a multi-item instrument ( Fayers \& Machin, 2000).

The only significant difference identified through the PedsQL ${ }^{\mathrm{TM}}$ analysis was that FA children reported better QoL in the physical functioning domain. This is positive, as it suggests that not all FA children experience difficulties in physical functioning and that they have similar QoL to non-FA children in other domains, contrary to previous research (Sicherer et al., 2001). This is also in contrast to research by the scale's developers, in a US population, which found that healthy children had the highest QoL in all domains, compared to children with chronic and acute illnesses (Varni et al., 2001). However, these results may be hindered by the questionnaire. Like previous research in a German sample of children with epilepsy and cancer (Felder-Puig et al., 2004), and as found in the parents questionnaire in the present study, many items from the PedsQL ${ }^{\mathrm{TM}}$ were slightly skewed towards the positive ratings of QoL, with some ceiling effects. Further research on this questionnaire in a UK sample, assessing item distribution, including floor and ceiling effects, would be useful.

\section{'Photovoice' analysis}

For most families with FA, the FA dominated their QoL, particularly in the food and drink, health, and education domains. The greatest impact on QoL in the education category was restrictions forced upon children in terms of school lunches. Research has identified that allergic reactions do occur in schools (Sampson et al., 1992 and Sicherer et al., 2001). The present study suggests that although this may be a risk, it should be evaluated in terms of the negative impact on QoL that some children face by being excluded from mealtimes with their peers. Advising US allergists, Young, Munoz-Furlong, and Sicherer (2009), suggest that children should not be separated from their friends during school lunches and that if 'allergen-safe' tables and classrooms are used, friends with safe lunches should also be allowed to sit at the table. The present study has found that this is not being implemented in some schools in the UK and the segregation and restrictions on school lunches had a negative impact on both the FA child and their parent's QoL. This is an issue that needs to be addressed and incorporated into guidelines for schools. The 
uncertainty regarding accidental allergic reactions may also be contributing to the impact of FA in the educational setting. Most allergic reactions occur away from home and are unpredictable (Pumphrey, 2004) and children may worry about the reaction of school friends or feeling self-consious if they have an accidental food-allergic reaction at school (DunnGalvin, Gaffney, \& O'B Hourihane, 2009).

Interestingly, not all FA children and their parents took photographs in the food/drink category and those that were taken reflected both positive and negative factors. Like Avery et al. (2003), the present study found that FA children took photographs relating to eating out and that most of these were positive. On the other hand, FA parents reported difficulties in eating out and frequently reported this as influencing their QoL negatively. This difference between FA children and their caregivers may be because adults protect their children from the difficulties they encounter, or that caregivers only allow their children to eat in places which they know to be safe.

Overall the study highlighted that the impact of FA on QoL is variable, with some families being more affected than others. All categories identified through the present study correlated with previously documented QoL domains apart from food/drink. This highlights one of the limitations of using generic QoL questionnaires, that is, they may omit factors that are salient to a specific population (Rapley, 2003). Particularly for FA parents, issues relating to food and concern over the health of their child were pertinent issues which affected QoL. These were not identified by the generic QoL scale, which only addressed the individual's own health and did not ask about food/drink. This highlights the importance of using FA specific QoL measures that are now available (DunnGalvin et al., 2008).

The present study found evidence to support the idea that caring for a child with a FA has a great impact on QoL with the burden of responsibility of the day-to-day management and emotional strain of the FA falling on the parent rather than the child. This supports findings by King et al. (2009) who found that mothers of children with a peanut allergy suffered high levels of stress and anxiety and had poorer overall QoL than the father. The lack of impact reported by the children in the present study may be due to their age. Parents may be adopting a protecting role, shielding their children from the impact of food allergies; and children at this age may not yet have begun to manage their own allergies. As children grow older, become more independent and develop a greater sense of identity, they may perceive a greater impact of FA on their lives. DunnGalvin et al. (2009) reported that food allergic children from the age of 12 years begin to feel different to others and by early teens children feel that food allergy has a much stronger impact on their lives. Alternatively, it may be that children have adapted to their illness and research suggests that people with chronic conditions commonly minimise the effects that their condition has on daily life (Falvo, 1999). It is therefore important to consider the developmental stage a child is at when examining the impact of FA on quality of life.

The limitations of this research should be considered when evaluating the study findings. One of the issues of studies combining quantitative and qualitative methodologies is the difference in sample sizes needed for these approaches. In the present study, the sample size was appropriate for qualitative analysis and although the response rate was lower than hoped, saturation point was reached during coding. However, for the questionnaire part of the study the sample size was very low reducing the power of the tests used. Further research looking at the differences in quality of life in food allergic and healthy groups are needed in order to examine the replicablility of these findings.

It is possible that the variability in the effect that FA had on QoL in this study was, at least in part, as a result of opening the inclusion criteria to include FA to any food. The broader sample makes it more difficult to identify whether allergies to different foods influenced QoL in different ways and potentially confounded the results. However, in reality, children often have multiple food allergies and the most common food allergies in children are to milk and eggs as well as peanuts (Bindslev-Jensen, 1998), so arguably the sample in the present study is a better representation of FA children, from the general population, than previous research restricted to children with peanut allergies. Similarly, the sample included families with additional atopic conditions which may have had an additional impact on QoL. As many people with food allergy also suffer from other atopic conditions future research could focus on how the interaction of these conditions impacts on QoL. 
A further restriction on the participant sample was that all FA families were recruited from a support group organisation for people at risk of anaphylaxis. Therefore, the sample contained a large number of children with severe FA. It may be that QoL is different for families living with mild/moderate FA; further research on this population would be useful. In addition, it is possible that the sample from the Anaphylaxis Campaign were well educated about their child's allergy and had already received much support about the management and treatment of their illness and thus the FA did not impact on their lives as much as it could. Nevertheless, the variability in responses, even from this self-selecting group, does suggest that it would be useful to assess QoL in clinic settings, to establish which families are in need of additional support and/or information to manage their child's FA.

\section{Conclusion}

This study has found that children were able to assess their own QoL using child friendly methods. Carrying medications, difficulties in eating school lunches, and restrictions on favourite foods negatively influenced QoL for FA children. Caring for a child with FA also impacted on the QoL of the parent. Overall QoL and QoL in social relationships were lower for FA parents than non-FA control parents. Eating out, shopping, and concern about the health of their child negatively influenced FA parents' QoL to a much greater extent than control parents. Using a mixed methods approach such as this has enabled greater insight into the effects of QoL on the daily lives of FA families and highlights the different ways in which children and their parents view their FA management. These different views should be taken into account when conducting similar studies in the future.

\section{References}

T. Arvola, A. Tahvanainen, E. Isolauri

Concerns and expectations of parents with atopic infants

Pediatric Allergy and Immunology, 11 (2000), pp. 183-188

N.J. Avery, R.M. King, S. Knight, J.O. Hourihane

Assessment of quality of life of children with a peanut allergy

Pediatric Allergy and Immunology, 14 (2003), pp. 378-382

C. Bindslev-Jensen

$A B C$ of allergies. Food allergy

British Medical Journal, 316 (1998), p. 1299

A.J. Cummings, R.C. Knibb, M. Erlewyn-Lajeunesse, R.M. King, G. Roberts, J.S. Lucas

Management of nut allergy influences quality of life and anxiety in children and their mothers

Pediatric Allergy and Immunology, 21 (2010), pp. 586-594

A.J. Cummings, R.C. Knibb, R.M. King, J.S. Lucas

The psychosocial impact of food allergy and food hypersensitivity in children, adolescents and their families. A review Allergy, 65 (2010), pp. 933-945

A. DunnGalvin, B.M. Flokstra-de Blok, A.W. Burks, A.E. Dubois, J.O. Hourihane

Food allergy quality of life questionnaire for children aged 0-12 years. Content, construct, and cross-cultural validity

Clinical and Experimental Allergy, 38 (2008), pp. 977-986

A. DunnGalvin, A. Gaffney, J. O’B Hourihane

Developmental pathways in food allergy. A new theoretical framework

Allergy, 64 (2009), pp. 560-568

\section{Eiser, R. Morse}

The measurement of quality of life in children. Past and future perspectives

Journal of Developmental and Behavioural Pediatrics, 22 (2001), pp. 1-9

D.R. Falvo

Medical and psychosocial aspects of chronic illness and disability

Aspen Publishers, Inc., Maryland (1999) 
P.M. Fayers, D. Machin

Quality of life: Assessment, analysis and interpretation

Wiley, Chichester (2000)

R. Felder-Puig, E. Frey, K. Proksch, J.W. Varni, H. Gadner, R. Topf

Validation of the German version of the Pediatric Quality of Life Inventory ${ }^{\mathrm{TM}}$ (PedsQL ${ }^{\mathrm{TM}}$ ) in childhood cancer patients off treatment and children with epilepsy

Quality of Life Research, 13 (2004), pp. 223-234

B.M. Flokstra-de Blok, A.E. Dubois, B.J. Vlieg-Boerstra, J.N. Oude Elberink, H. Raat, A. DunnGalvin et al. Health-related quality of life of food allergic patients. Comparison with the general population and other diseases Allergy, 65 (2010), pp. 238-244

B.M. Flokstra-de Blok, J.L. van der Velde, B.J. Vlieg-Boerstra, J.N. Oude Elberink, A. DunnGalvin, J.O. Hourihane et al. Health-related quality of life of food allergic patients measured with generic and disease-specific questionnaires

Allergy, 65 (2010), pp. 1031-1038

M.H. Gowland

Food allergen avoidance. Risk assessment for life

Proceedings of the Nutrition Society, 61 (2002), pp. 39-43

\section{C.L. Hanson}

Quality of life in families of youths with chronic conditions

H.M. Koot, J.L. Wallander (Eds.), Quality of life in child and adolescent illness: Concepts, methods, and findings, Brunner-Routledge, Hove (2001), pp. 181-209

\section{J.O. Hourihane}

The threshold concept in food safety and its applicability to food allergy

Allergy, 56 (2001), pp. 86-90

P. Jirojanakul, S. Skevington

Developing a quality of life measure for children aged 5-8 years

British Journal of Health Psychology, 5 (2000), pp. 299-321

R.M. King, R.C. Knibb, J.O. Hourihane

Impact of peanut allergy on quality of life, stress and anxiety in the family

Allergy, 64 (2009), pp. 461-468

K.A. Neuendorf

The content analysis guidebook

Sage, London (2001)

M.N. Primeau, R. Kagan, L. Joseph, H. Lim, C. Dufresne, C. Duffy et al.

The psychological burden of peanut allergy as perceived by adults with peanut allergy and the parents of peanut allergic children

Clinical and Experimental Allergy, 30 (2000), pp. 1135-1143

R. Pumphrey

Anaphylaxis. Can we tell who is at risk of a fatal reaction?

Current Opinion in Allergy and Clinical Immunology, 4 (2004), pp. 285-290

M. Rapley

Quality of life research: A critical introduction

Sage, London (2003)

E. Sales

Family burden and quality of life

Quality of Life Research, 12 (2003), pp. 33-41 
New England Journal of Medicine, 327 (1992), pp. 380-381

S.H. Sicherer, S.A. Noone, A. Munoz-Furlong

The impact of childhood food allergy on quality of life

Annals of Allergy, Asthma and Immunology, 87 (2001), pp. 461-464

S.M. Skevington, M. Lotfy, K.A. O'Connell

The World Health Organization's WHOQOL-BREF quality of life assessment. Psychometric properties and results of the international field trial.

A report from the WHOQOL Group

Quality of Life Research, 13 (2004), pp. 299-310

E.E. Springston, J. Shulruff, B. Smith, X. Wang, J. Pongracic, J. Holl et al.

Variations in quality of life among caregivers of food-allergic children

Annals of Allergy, Asthma and Immunology, 105 (2010), pp. 287-294

J.W. Varni, M. Seid, P.S. Kurtin

The PedsQL ${ }^{\mathrm{TM}}$ 4.0. Reliability and validity of the Pediatric Quality of Life Inventory ${ }^{\mathrm{TM}}$ Version 4.0 Generic Core Scales in healthy and patient populations

Medical Care, 39 (2001), pp. 800-812

Varni, J. W. (2004). The PedsQL' http://www.pedsql.org/score.html

J.L. Wallander

Theoretical and developmental issues in quality of life for children and adolescents

H.M. Koot, J.L. Wallander (Eds.), Quality of life in child and adolescent illness: Concepts, methods, and findings, Brunner-Routledge, Hove (2001), pp. 23-48

WHOQOL Group (The)

The World Health Organisation Quality of Life Assessment (WHOQOL). Development and general psychometric properties Social Science and Medicine (1998), pp. 1569-1585

WHOQOL Group (The)

WHOQOL-BREF: Introduction, administration, scoring and generic version of the assessment

WHO, Geneva (1996)

M.C. Young, A. Munoz-Furlong, S.H. Sicherer

Management of food allergies in schools. A perspective for allergists

Journal of Allergy and Clinical Immunology, 124 (2009), pp. 175-182 